\title{
Activation of Coagulation and Fibrinolysis in Acute Respiratory Distress Syndrome: A Prospective Pilot Study
}

\author{
Agnese Ozolina ${ }^{1,2 *}$, Marina Sarkele,2,3, Olegs Sabelnikovs ${ }^{2,3}$, Andrejs Skesters ${ }^{4}$, \\ Inta Jaunalksne ${ }^{5}$, Jelena Serova ${ }^{5}$, Talis levins ${ }^{1}$, Lars J. Bjertnaes ${ }^{6}$ and Indulis Vanags ${ }^{2,3}$ \\ 'Department of Cardiac Surgery, Pauls Stradins Clinical University Hospital, Riga, Latvia, ${ }^{2}$ Riga Stradins University, Riga, \\ Latvia, ${ }^{3}$ Department of Anesthesiology and Intensive Care Unit, Pauls Stradins Clinical University Hospital, Riga, Latvia, \\ ${ }^{4}$ Laboratory of Biochemistry, Riga Stradins University, Riga, Latvia, ${ }^{5}$ Clinical Immunology Centre, Pauls Stradins Clinical \\ University Hospital, Riga, Latvia, ${ }^{6}$ Anesthesia and Critical Care Research Group, Department of Clinical Medicine, Faculty of \\ Health Sciences, University of Tromsø, Tromsø, Norway
}

OPEN ACCESS

Edited by:

César Aldecoa,

Hospital Universitario

Río Hortega, Spain

Reviewed by:

Lieuwe D. J. Bos,

University of Amsterdam,

Netherlands

Vsevolod V. Kuzkov,

Northern State Medical

University, Russia

*Correspondence:

Agnese Ozolina

agnese.ozolina@icloud.com

Specialty section:

This article was submitted

to Intensive Care Medicine

and Anesthesiology,

a section of the journal

Frontiers in Medicine

Received: 22 July 2016 Accepted: 15 November 2016 Published: 28 November 2016

Citation:

Ozolina A, Sarkele M, Sabelnikovs O, Skesters A, Jaunalksne I, Serova J, levins $T$, Bjertnaes $L J$ and Vanags I (2016) Activation of Coagulation and

Fibrinolysis in Acute Respiratory

Distress Syndrome: A Prospective Pilot Study.

Front. Med. 3:64.

doi: 10.3389/fmed.2016.00064
Introduction: Coagulation and fibrinolysis remain sparsely addressed with regards to acute respiratory distress syndrome (ARDS). We hypothesized that ARDS development might be associated with changes in plasma coagulation and fibrinolysis. Our aim was to investigate the relationships between ARDS diagnosis and plasma concentrations of tissue factor (TF), tissue plasminogen activator (t-PA), and plasminogen activator inhibitor-1 (PAl-1) in mechanically ventilated patients at increased risk of developing ARDS.

Materials and methods: We performed an ethically approved prospective observational pilot study. Inclusion criteria were patients with $\mathrm{PaO}_{2} / \mathrm{FiO}_{2}<300 \mathrm{mmHg}$ admitted to the intensive care unit (ICU) for mechanical ventilation for $24 \mathrm{~h}$, or more, because of one or more disease conditions associated with increased risk of developing ARDS. Exclusion criteria were age below 18 years; cardiac disease. We sampled plasma prospectively and compared patients who developed ARDS with those who did not using descriptive statistics and chi-square analysis of baseline demographical and clinical data. We also analyzed plasma concentrations of TF, t-PA, and PAl-1 at inclusion $\left(T_{0}\right)$ and on third $\left(T_{3}\right)$ and seventh day $\left(T_{7}\right)$ of the ICU stay with non-parametric statistics inclusive their sensitivity and specificity associated with the development of ARDS using receiver operating characteristic curve analysis. Statistical significance: $p<0.05$.

Results: Of 24 patients at risk, 6 developed mild ARDS and 4 of each moderate or severe ARDS, respectively, $3 \pm 2$ (mean \pm SD) days after inclusion. Median plasma concentrations of TF and PAl-1 were significantly higher at $T_{7}$ in patients with ARDS, as compared to non-ARDS. Simultaneously, we found moderate correlations between plasma concentrations of TF and PAI-1, TF and $\mathrm{PaO}_{2} / \mathrm{FiO}_{2}$, and positive end-expiratory pressure and TF. TF plasma concentration was associated with ARDS with $71 \%$ sensitivity and 100\% specificity, a cut off level of $145 \mathrm{pg} / \mathrm{ml}$ and AUC 0.78, $p=0.02$. PAl-1 displayed $64 \%$ sensitivity and $100 \%$ specificity with a cut off concentration of $117.5 \mathrm{pg} / \mathrm{ml}$ and AUC 0.77, $p=0.02$. t-PA did not change significantly during the observation time. 
Conclusion: This pilot study showed that increased plasma concentrations of TF and PAl-1 might support ARDS diagnoses in mechanically ventilated patients after 7 days in ICU.

Keywords: acute respiratory distress syndrome, lung injury, plasminogen activator inhibitor-1, tissue factor, tissue plasminogen activator, ventilator-associated lung injury

\section{INTRODUCTION}

Acute respiratory distress syndrome (ARDS) represents a severe respiratory failure characterized by hypoxia and nonhydrostatic pulmonary edema with bilateral opacities on frontal chest radiographs. Patients with ARDS require treatment in the intensive care unit (ICU) for respiratory support with continuous positive airway pressure (CPAP) or positive end-expiratory pressure (PEEP) of $5 \mathrm{cmH}_{2} \mathrm{O}$ or higher in combination with mechanical ventilation (1-3). Usually, ARDS occurs within 1 week of a triggering event $(4,5)$ and might be the result of a direct lung injury, as pneumonia, or an indirect lung injury, like septic shock (6-8).

Incidence of ARDS displays large geographical differences (9, 10). A recent worldwide multicenter study showed that $10.4 \%$ of those admitted to participating ICUs and $23.4 \%$ of those requiring mechanical ventilation exhibited ARDS criteria. Hospital mortality varied among 34.9, 40.3, and $46.1 \%$ for patients with, respectively, mild, moderate, and severe ARDS (11). This is consistent with mortality rates published by previous investigators $(10,12)$. Thus, despite a varying incidence, overall mortality from ARDS has not changed substantially during the last decade $(13,14)$. Consequently, identification of patients at risk should be prioritized with the aim to diagnose ARDS earlier, as recently proposed (15).

Several studies have focused on injury to the lung parenchyma, which then causes deteriorated gas exchange in ARDS (16-18). In contrast, changes in homeostasis of coagulation and fibrinolysis have been sparsely investigated despite that their role in the evolution of this disease is beyond doubt. Investigators recently described a role in the interaction between lung inflammation and the coagulation and the fibrinolytic systems for the commencement of ARDS $(19,20)$.

Inflammation modulates blood coagulation by activating C-reactive protein, which stimulates monocytes and alveolar macrophages to generate tissue factor $(\mathrm{TF})(5,21)$, and endothelial cells to produce plasminogen activator inhibitor-1 (PAI-1) (22). The combined effects can lead to disseminated intravascular coagulation (DIC) including formation of intravascular micro-thrombi and intra-alveolar fibrin deposits (5, 22-24), subsequently increasing both dead-space ventilation and intrapulmonary shunting, both characteristic features of ARDS (23). Consequently, we speculated that the circulatory level of TF, and the balance between inhibitors and activators of fibrinolysis, could be of help for the diagnosing of ARDS (21).

In mechanically ventilated patients, whom we considered to be at increased risk of developing ARDS, we aimed to perform a pilot study to explore whether associations exist between diagnosis of ARDS and the plasma concentration of TF as the primary endpoint, and ARDS and plasma levels of PAI-1 and tissue plasminogen activator ( $\mathrm{t}-\mathrm{PA}$ ), respectively, as secondary endpoints.

\section{MATERIALS AND METHODS}

\section{Patients Eligible for the Study}

Between November 2014 and February 2015, we transferred 33 patients to the ICU of Pauls Stradins Clinical University Hospital (PSCU), Riga, Latvia, for mechanical ventilation because we considered them to be at increased risk of developing ARDS. After the Medical Ethics Committee of PSCU had approved the study protocol and the informed consent form (No. 18204-5 L), we screened the patients for suitability to participate in a prospective observational pilot study of the influence of plasma coagulation and fibrinolysis on the emergence of ARDS. We obtained informed consent from each one's legal guardian prior to inclusion for those who were eligible for participation in the study.

According to their disease conditions, the patients were treated either with non-invasive pressure-controlled mechanical ventilation (NIV), or endotracheal intubation and mechanical ventilation with CPAP $\geq 5 \mathrm{cmH}_{2} \mathrm{O}$, or PEEP $\geq 5 \mathrm{cmH}_{2} \mathrm{O}$ over 24 h or more using Servo-I ventilators (Maquet Getinge, Group, Rastatt, Germany). The patients received antibiotics, as required, based on the results of microbiological cultures and antibiotic resistance testing.

\section{Inclusion Criteria}

We included patients with $\mathrm{PaO}_{2} / \mathrm{FiO}_{2}<300 \mathrm{mmHg}$ and with an increased risk of developing ARDS (23) because of one or more ARDS predisposing conditions causing either direct or indirect lung injury. The former included pneumonia or aspiration of gastric contents into the airways; the latter comprised of sepsis, acute pancreatitis, DIC, burns, drug overdose or trauma with circulatory shock, and massive blood transfusions, as outlined by previous investigators (23). The patients did not fulfill all the required ARDS criteria at the time of inclusion (2). Those who fulfilled ARDS criteria within 1 week of the stay in ICU were randomized to an ARDS group, the others to a non-ARDS group.

We divided those who fulfilled the clinical, radiological, and cardiologic criteria of ARDS into mild, moderate, or severe ARDS with the characteristics listed below. Mild ARDS: $200 \mathrm{mmHg}<\mathrm{PaO}_{2} / \mathrm{FiO}_{2} \leq 300 \mathrm{mmHg}$, CPAP or PEEP $\geq 5 \mathrm{cmH}_{2} \mathrm{O}$; moderate ARDS: $100 \mathrm{mmHg}<\mathrm{PaO}_{2} / \mathrm{FiO}_{2} \leq 200 \mathrm{mmHg}$, PEEP $\geq 5 \mathrm{cmH}_{2} \mathrm{O}$; and severe ARDS: $\mathrm{PaO}_{2} / \mathrm{FiO}_{2} \leq 100 \mathrm{mmHg}, \mathrm{PEEP}$ $\geq 5 \mathrm{cmH}_{2} \mathrm{O}$, according to the definition (2). 


\section{Exclusion Criteria}

We excluded patients below 18 years of age, patients receiving mechanical ventilation with no ARDS predisposing condition, patients not fulfilling the study protocol, and patients from whom we did not get consent for participation. We also excluded patients after transthoracic echocardiography (ECHO) displaying ejection fraction $(\mathrm{EF})<50 \%$ and right ventricular systolic pressure $>35-40 \mathrm{mmHg}$, and with a pulmonary artery occlusion pressure $>18 \mathrm{mmHg}$, as measured if a Swan Ganz catheter was in place.

\section{Study Protocol}

We determined plasma concentrations of TF, t-PA, and PAI-1 at enrollment $\left(T_{0}\right)$ and on the third day $\left(T_{3}\right)$ and the seventh day $\left(T_{7}\right)$ of the ICU stay. Moreover, we noticed ventilator settings including $\mathrm{FiO}_{2}$, tidal volume, airway plateau pressure, and PEEPlevels that were titrated by means of the transpulmonary pressure-volume curve displayed on the Servo-I ventilator, and the resulting readings of $\mathrm{PaO}_{2} / \mathrm{FiO}_{2}$ and lung mechanical parameters from every subject at the time of collection of plasma samples. We also recorded number of days on mechanical ventilation, ventilator-free days at day 30 of the stay in ICU, and the 30-day mortality rate.

We observed the patients in ICU for 1 week and compared plasma concentrations of coagulation and fibrinolysis biomarkers between those who developed ARDS and those who did not develop the disease (non-ARDS), as based on recent criteria (2). Demographic and clinical data and direct and indirect ARDS risk factors were retrieved from the medical records of the patients. The severity of disease was scored at inclusion using the sequential organ failure assessment (SOFA) and the acute physiology and chronic health evaluation (APACHE II) scores. In addition, we calculated lung injury prediction score (LIPS) for every patient at the day of inclusion $\left(T_{0}\right)$.

\section{Determination of Biomarkers of Coagulation and Fibrinolysis}

We collected blood from a peripheral vein into heparin tubes that we cool centrifuged (ELMI CM-6MT ${ }^{\circledR}$, USA) for $5 \mathrm{~min}$ at $3,000 \times g$. The plasma supernatant was removed from the spun samples and frozen at $-70^{\circ} \mathrm{C}$ until the time of analysis. Human TF (CD264) with a normal range of from 12.5 to $400 \mathrm{pg} / \mathrm{ml}$ was quantitatively assessed by means of an enzyme-linked immunosorbent assay (ELISA) test $\left(\mathrm{Abcam}^{\circledR}, \mathrm{UK}\right)$. Fibrinolysis markers, t-PA (normal range 15.6-1,000 pg/ml), and PAI-1 (normal range $78-5,000 \mathrm{pg} / \mathrm{ml}$ ) were analyzed with the same method using the platinum ELISA kit (eBioscience ${ }^{\circledR}$, USA).

In parallel, we analyzed standard coagulation tests (SCT) such as prothrombin index (PI), international normalized ratio (INR), and fibrinogen plasma concentration, as well as platelet count (PLT), which was analyzed by means of a Beckman Coulter LH 750 Hematology Analyzer (Beckman Coulter International SA, Switzerland). Coulter LH 750 uses impedance technology to measure PLT count (normal range $\left.150-450 \times 10^{9} / 1\right)$. Fibrinogen plasma concentration was analyzed in citrated plasma (Multifibren U reagent, Siemens Healthcare Diagnostics, USA). The reference value is $1.8-3.6 \mathrm{~g} / \mathrm{l}$. PI was analyzed using a prothrombin complex assay (Lyophilized Dade ${ }^{\circledR}$ and Innovin ${ }^{\circledR}$ reagent, Siemens
Healthcare Diagnostics, USA). PI normal range is $70-120 \%$. APTT was analyzed in citrated human plasma (Pathrombin ${ }^{\star}$ SL reagent, Siemens Healthcare Diagnostics, USA). APTT normal range is $26-36 \mathrm{~s}$.

\section{Statistical Analysis and Sample Size Calculations}

Statistical analyses were performed using the SPSS Statistics (Chicago, IL, USA, Version 20). We compared ARDS patients and non-ARDS patients and survivors and non-survivors of the disease. Data distribution was assessed using Shapiro-Wilk test or Kolmogorov-Smirnov test, as appropriate. We used descriptive statistics for analysis of baseline demographics and clinical data, and Chi-square or Mann-Whitney rank sum test to compare the difference between groups. If the $F$ value was greater than critical, repeated measures ANOVA was followed by Holm-Sidak's post hoc test for pairwise multiple comparisons to check for intragroup differences. For non-normally distributed data, the results were presented as boxes with median and interquartile range (IQR; 25th-75th percentile), including vertical error bars for the 10 and $90 \%$ lowest and highest values, respectively. Correlations were presented as Spearman's $r_{\mathrm{s}}$ (25). We performed receiver operating characteristic (ROC) curve analysis of coagulation and fibrinolytic biomarkers including calculations of area under the curve (AUC) for TF and PAI-1 to demonstrate their efficiency in supporting ARDS diagnosis. Spearman's correlation coefficient also was calculated to analyze the relationships between coagulation/fibrinolysis markers and ventilation parameters. Statistical significance was defined as $p<0.05$. When required, we calculated sample sizes needed to reach significant differences $(p<0.05)$ in plasma concentrations of TF, PAI-1, and t-PA at a power of $80 \%$ between the ARDS group and the non-ARDS group.

\section{RESULTS}

\section{Clinical Course}

As depicted in Figure 1, we assessed 33 mechanically ventilated patients, considered to be at risk of developing ARDS, for suitability to the study. We excluded seven patients who died before they fulfilled all the requirements of the study protocol, and two patients from whom we did not get consent for participation.

Table 1 displays demographic characteristics and clinical variables of 24 patients ( 21 men and 3 women) fulfilling inclusion criteria and all requirements of the protocol encompassing comorbidities, conditions predisposing for ARDS, severity scores, and standard coagulation variables. Pneumonia (46\%) was the most common underlying disease. However, neither ARDS nor non-ARDS patients displayed significant intergroup differences with regard to demographic data, comorbidities, or predisposing conditions. Patients diagnosed with ARDS had significantly higher baseline values of SOFA, APACHE II, and LIPS scores. Moreover, the ARDS group showed significant differences in systemic coagulation (elevated INR, lower PLT, and fibrinogen plasma levels), but no clinical signs of DIC. The mean time for developing ARDS was $3 \pm 2$ days after inclusion. Six patients developed mild ARDS, whereas moderate or severe ARDS occurred within 1 week of the inclusion in four patients 


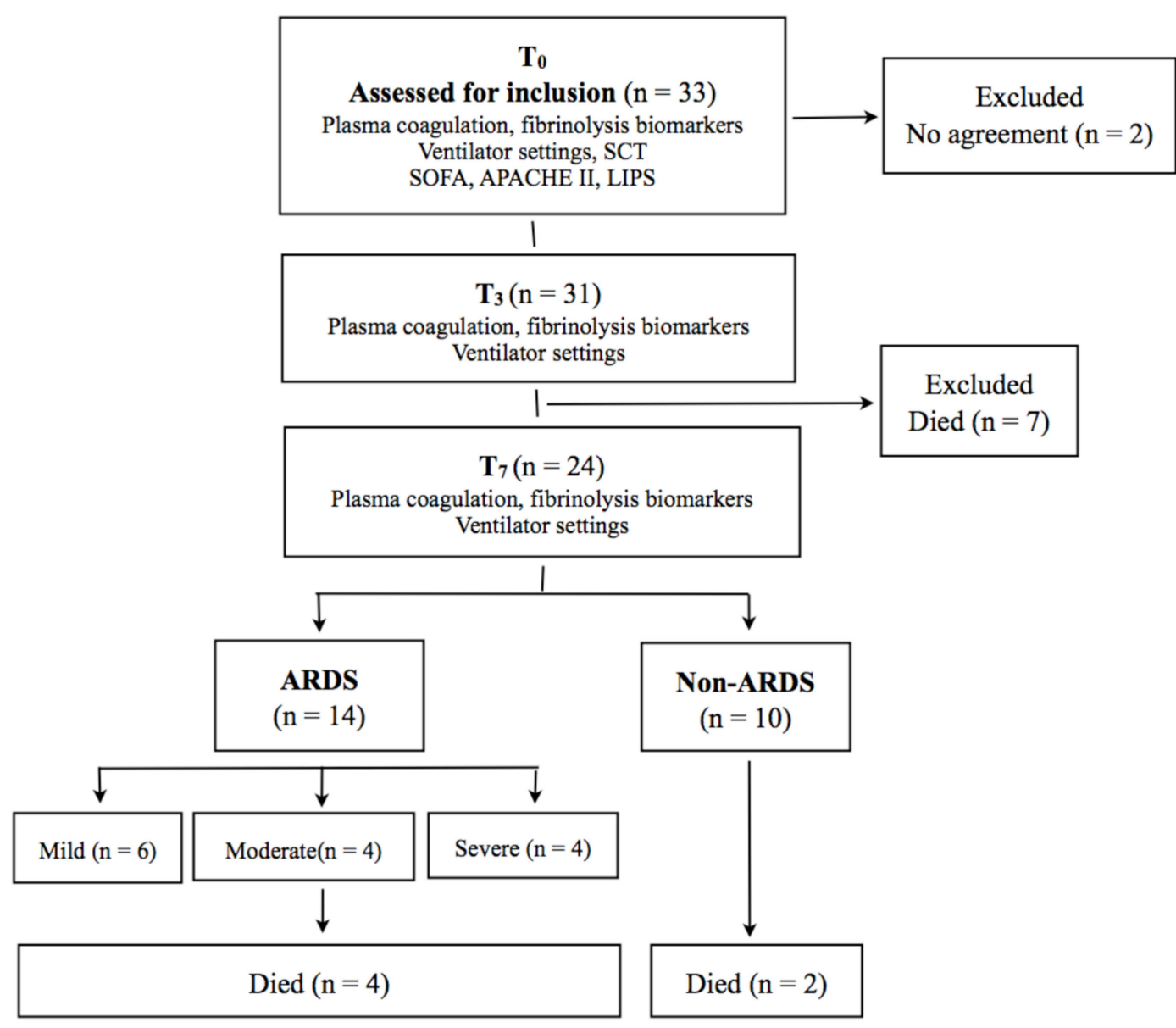

FIGURE 1 | Mechanically ventilated patients considered for eligibility to a prospective pilot study. Twenty-four patients fulfilled the study protocol. SCT, standard coagulation tests; APACHE II, acute physiology and chronic health evaluation II; SOFA, sequential organ failure assessment; LIPS, lung injury prediction score; ARDS, acute respiratory distress syndrome.

each, thus constituting in all 58\% of the population at risk. Days in ICU and ventilator-free days at day 30 displayed no intergroup differences. Total hospital mortality and mortality at day 30, reached 25\%, but displayed no significant differences between the groups.

Table 2 displays ventilation parameters and $\mathrm{PaO}_{2} / \mathrm{FiO}_{2}$ at $T_{0}$, $T_{3}$, and $T_{7}$. Tidal volumes were significantly smaller at $T_{0}$ and $T_{7}$ in ARDS patients, as compared to the non-ARDS group. Despite that plateau pressures were significantly higher in the ARDS group at $T_{3}$ and $T_{7}$, and PEEP also was significantly raised at the latter time point, these ventilator settings did not prevent a significant fall in $\mathrm{PaO}_{2} / \mathrm{FiO}_{2}$ in comparison with the non-ARDS group.

\section{Comparison of Coagulation and Fibrinolysis in ARDS vs. Non-ARDS Patients}

Plasma concentrations of TF (Figure 2A) remained within normal range with no significant difference between ARDS and non-ARDS group at $T_{0}(p=0.229)$, but displayed marked intergroup differences at $T_{3}(p=0.043)$ and $T_{7}(p=0.022)$. The ARDS group also showed intragroup differences in TF plasma concentrations between the highest value at $T_{7}$ in comparison with those at $T_{0}$ and $T_{3}$, respectively $(p<0.001)$. In contrast, we found no significant intragroup differences in the plasma levels of TF in the non-ARDS group.

Plasma levels of PAI-1 (Figure 2B) showed no intergroup differences at $T_{0}$ and $T_{3}$, but increased at $T_{7}$ in the ARDS group, as compared with non-ARDS group $(p=0.026)$. Plasma concentration of PAI- 1 at $T_{7}$ in the ARDS group was also increased in comparison with intragroup values at $T_{0}(p=0.016)$ and $T_{3}$ $(p=0.017)$, but not no significant difference was found between the latter concentrations. Plasma levels of t-PA fell at $T_{3}$ (Figure 2C), but we noticed no significant differences within or between the groups. At $T_{3}$, plasma concentration of t-PA was $295 \pm 85 \mathrm{pg} / \mathrm{ml}$ $($ mean $\pm \mathrm{SD})$ in ARDS survivors vs. $516 \pm 160 \mathrm{pg} / \mathrm{ml}$ in ARDS non-survivors, but the difference did not reach significance due to low sample size.

We found significant correlations between plasma concentrations of TF and PAI-1 $(r=0.53 ; p=0.008)$, TF and $\mathrm{PaO}_{2} / \mathrm{FiO}_{2}$ 
TABLE 1 | Baseline demographic characteristics, ARDS predisposing factors, and mortality.

\begin{tabular}{|c|c|c|c|c|}
\hline Demographic data & Totally, $n=24$ & ARDS, $n=14$ & Non-ARDS, $n=10$ & $p$-Value \\
\hline Age, years & $54 \pm 17$ & $55 \pm 15$ & $53 \pm 19$ & 0.7 \\
\hline Women, $n(\%)$ & $3(12)$ & $2(14)$ & $1(10)$ & 0.8 \\
\hline $\mathrm{BMI}$ & $26.4 \pm 4.6$ & $24.7 \pm 2.8$ & $27.2 \pm 3.2$ & 0.4 \\
\hline \multicolumn{5}{|l|}{ Comorbidities } \\
\hline Hypertension, $n$ (\%) & $7(29)$ & $4(29)$ & $3(30)$ & 0.1 \\
\hline Diabetes mellitus, $n$ (\%) & $4(17)$ & $2(14)$ & $2(20)$ & 0.6 \\
\hline COPD, $n(\%)$ & $3(12)$ & $1(7)$ & $2(20)$ & 0.4 \\
\hline \multicolumn{5}{|c|}{ Conditions predisposing for ARDS } \\
\hline Pneumonia, $n(\%)$ & $11(46)$ & $6(42)$ & $5(50)$ & 0.7 \\
\hline Sepsis, $n(\%)$ & $7(29)$ & $4(29)$ & $3(30)$ & 0.9 \\
\hline Pancreatitis, $n$ (\%) & $5(21)$ & $4(29)$ & $1(10)$ & 0.3 \\
\hline Massive transfusions, $n(\%)$ & $1(4)$ & 0 & $1(10)$ & 0.2 \\
\hline \multicolumn{5}{|l|}{ Severity scores } \\
\hline SOFA, median (range) & $4(3.4-6.1)$ & $5(4.6-7.2)$ & $3(2.1-4.9)$ & 0.04 \\
\hline APACHE II, median (range) & $23(19-28)$ & $25(22-28)$ & $21(19-24)$ & 0.03 \\
\hline LIPS median (range) & $5.8(0.3-13)$ & $8.1(3-13)$ & $4.9(0.3-9)$ & 0.01 \\
\hline \multicolumn{5}{|l|}{ Coagulation status } \\
\hline INR & $1.18 \pm 0.4$ & $1.2 \pm 0.6$ & $1.12 \pm 0.15$ & 0.03 \\
\hline PLT (109/l) & $172 \pm 56$ & $136 \pm 47$ & $213 \pm 31$ & 0.003 \\
\hline Fibrinogen (mg/dl) & $283 \pm 71$ & $261 \pm 96$ & $312 \pm 63$ & 0.02 \\
\hline \multicolumn{5}{|c|}{ ICU stay and mechanical ventilation } \\
\hline ICU days, $n$ & $12 \pm 5$ & $14 \pm 7$ & $11 \pm 4$ & 0.7 \\
\hline Ventilator-free days, $n$ & $5 \pm 3$ & $4 \pm 2$ & $7 \pm 4$ & 0.4 \\
\hline \multicolumn{5}{|l|}{ Mortality } \\
\hline Hospital, $n$ (\%) & $6(25)$ & $4(29)$ & $2(20)$ & 0.1 \\
\hline 30-day, $n(\%)$ & $6(25)$ & $4(29)$ & $2(20)$ & 0.1 \\
\hline
\end{tabular}

Data are presented as mean $\pm S D$, number ( $n$ ), or median (range).

APACHE II, chronic health evaluation score; ARDS, acute respiratory distress syndrome; BMI, body mass index; ICU, intensive care unit; INR, international normalized ratio; LIPS,

lung injury prediction score; $n$, number of patients; PLT, platelets; \%, percent; range, lowest and highest value; SOFA, sequential organ failure assessment score.

TABLE 2 | Ventilation variables at inclusion $\left(T_{0}\right)$ and at the third $\left(T_{3}\right)$ and seventh $\left(T_{7}\right)$ day of ICU stay in ARDS and non-ARDS patients.

\begin{tabular}{|c|c|c|c|c|c|}
\hline \multirow{2}{*}{$\begin{array}{l}\text { Ventilation } \\
\text { settings }\end{array}$} & \multicolumn{2}{|c|}{ ARDS, $n=14$} & \multicolumn{2}{|c|}{ Non-ARDS, $n=10$} & \multirow[t]{2}{*}{$p$-Value } \\
\hline & Median & IQR & Median & IQR & \\
\hline \multicolumn{6}{|c|}{ Tidal volume, I/min } \\
\hline$T_{0}$ & 8.6 & $7.1-9.2$ & 10.1 & $8.8-11.3$ & 0.01 \\
\hline$T_{3}$ & 7.8 & $6.7-8.9$ & 9.6 & $8.9-10.1$ & NS \\
\hline$T_{7}$ & 7.1 & $6.6-7.7$ & 8.7 & $8.3-9.0$ & 0.002 \\
\hline \multicolumn{6}{|c|}{ Plateau pressure, $\mathrm{cmH}_{2} \mathrm{O}$} \\
\hline$T_{0}$ & 29 & 22-35 & 24 & $21-27$ & NS \\
\hline$T_{3}$ & 30 & $23-36$ & 26 & $22-30$ & 0.002 \\
\hline$T_{7}$ & 27 & $21-34$ & 25 & $20-31$ & 0.012 \\
\hline \multicolumn{6}{|l|}{$\mathrm{PaO}_{2} / \mathrm{FiO}_{2}$} \\
\hline$T_{0}$ & 158.5 & $42-282$ & 183.2 & $74-348$ & NS \\
\hline$T_{3}$ & 193.5 & $94-362$ & 234 & $58-358$ & NS \\
\hline$T_{7}$ & 179.5 & $57-292$ & 284 & 80-356 & 0.03 \\
\hline \multicolumn{6}{|c|}{ PEEP, $\mathrm{cmH}_{2} \mathrm{O}$} \\
\hline$T_{0}$ & 7 & $5-11$ & 5 & $3-7$ & NS \\
\hline$T_{3}$ & 7 & $5-13$ & 5 & $4-7$ & NS \\
\hline$T_{7}$ & 7 & $5-13$ & 5 & $2-6$ & 0.006 \\
\hline
\end{tabular}

Data are presented as median and interquartile range (IQR).

ARDS, acute respiratory distress syndrome; $\mathrm{CmH}_{2} \mathrm{O}$, centimeter of water; $\mathrm{FiO}_{2}$, fraction of inspired oxygen; kg, kilograms; $\mathrm{ml}$, milliliters; $n$, number of patients; $\mathrm{PaO}_{2}$, partial pressure of oxygen in blood; PEEP, positive end-expiratory pressure; NS, not significant.

$(r=-0.65 ; p=0.001)$, and PEEP and TF $(r=0.55 ; p=0.005)$ at time $T_{7}$, as depicted in Figures 3A-C.

In ARDS patients, a ROC analysis (Figure 4) showed $71 \%$ sensitivity and $100 \%$ specificity for an association with $\mathrm{TF}$ at $T_{7}$ with a cut off value of $145 \mathrm{pg} / \mathrm{ml}$ [AUC $0.782 ; p=0.021 ; 95 \%$ confidence interval (95\%CI): 0.586-0.979]. We also found an association with PAI-1 determined at the same time point with a sensitivity and specificity of 64 and $100 \%$, respectively, with a cut off value of $117.5 \mathrm{pg} / \mathrm{ml}$ (AUC 0.775; $p=0.024,95 \% \mathrm{CI}$ : 0.584-0.966).

\section{DISCUSSION}

In this prospective pilot study, 14 out of total 24 patients considered to be at increased risk of ARDS, developed the disease within 7 days of commencement of mechanical ventilation. Patients diagnosed with ARDS had significantly higher baseline severity scores in comparison with the non-ARDS group, which could indicate a worse outcome. However, mortality did not differ significantly between groups (Table 1). In the ARDS group, $\mathrm{PaO}_{2} /$ $\mathrm{FiO}_{2}$ reached nadir on the seventh day of the ICU stay, in parallel with significant changes in variables of coagulation and fibrinolysis. In patients diagnosed with ARDS, plasma concentrations of TF and PAI-1 increased significantly at $T_{7}$, as compared with the non-ARDS group. The observation of increased TF is consistent with recent findings in patients with sepsis-induced ARDS (5). Therefore, our findings suggest a role for these biomarkers as supportive diagnostic tools during the emergence of ARDS.

Development of ARDS in a population at risk depends on both genetic predisposition and interaction between several biological pathways, including inflammation, coagulation, and fibrinolysis (19). We intentionally observed patients in the ICU for 1 week 

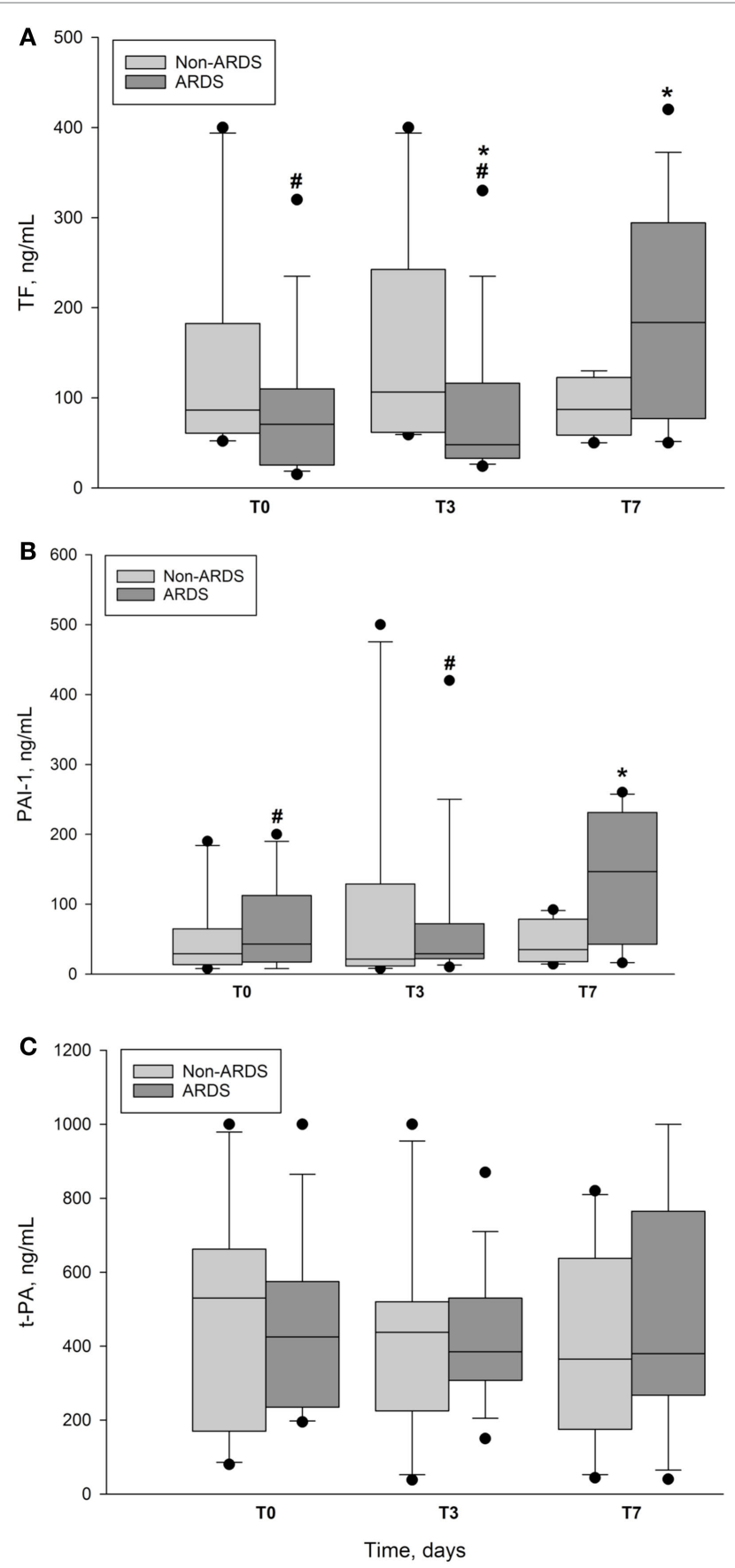

FIGURE 2 | Plasma concentrations of markers of coagulation and fibrinolysis in ARDS and non-ARDS patients. (A) Plasma concentration of tissue factor (TF) vs. time in ARDS group and non-ARDS group. Data presented as median and interquartile range with 10th and 90th percentiles error bars. $T_{0}$ is day of enrollment, $T_{3}$ and $T_{7}$, the third day and the seventh day of ICU stay. ${ }^{*}$ denotes intergroup $p=0.043$ at $T_{3}$ and $p=0.022$ at $T_{7}$. "denotes ARDS intragroup $p \leq 0.001$ between $T_{7}$ and $T_{0}$ and $T_{3}$, respectively. $\mathrm{pg} / \mathrm{ml}$, picogram per milliliter; closed circles represent outliers. (B) Plasma concentrations of plasminogen activator inhibitor-1 (PAI-1) vs. time in ARDS group and non-ARDS group. Data presented as median and interquartile range with 10th and 90th percentiles error bars. $T_{0}$ is day of enrollment, $T_{3}$ and $T_{7}$, the third day and the seventh day of ICU stay.

(Continued)

\section{FIGURE 2 | Continued}

*denotes intergroup $p=0.026$ at $T_{7}$. "denotes ARDS intragroup $p=0.016$ between $T_{7}$ and $T_{0}$ and $p=0.017$ between $T_{7}$ and $T_{3} . \mathrm{pg} / \mathrm{ml}$, picogram per milliliter; closed circles represent outliers. (C) Plasma concentration of tissue plasminogen activator (t-PA) vs. time in ARDS group and non-ARDS group. Data presented as median and interquartile range with 10th and 90th percentiles error bars. $T_{0}$ is day of enrollment, $T_{3}$ and $T_{7}$, the third day and the seventh day of ICU stay. No significant intergroup or intragroup differences; closed circles represent outliers.

after inclusion for biomarker detection. This was inspired by observational studies suggesting that the majority of patients are identified with ARDS within $72 \mathrm{~h}$ of the recognition of risk factors, and nearly all within 7 days of hospital stay $(22,25,26)$. However, in spite of our efforts to keep low tidal volumes and to titrate PEEP to a point above the lower inflection of the transpulmonary pressure-volume curve, yielding optimal oxygenation, both tidal volume and plateau pressure increased significantly at $T_{7}$, and we were not able to prevent $\mathrm{PaO}_{2} / \mathrm{FiO}_{2}$ from falling in the ARDS group. This is consistent with the derangements in oxygenation and lung mechanics in developing ARDS, as reported by other researchers $(2,14)$.

\section{ARDS and Biomarkers of Coagulation and Fibrinolysis}

Investigators have focused on different biomarkers of ARDS (3, $5,19,24)$, but so far, without significant preferences. Protein C plasma levels are reduced in patients with severe sepsis (26) and in victims of ARDS, due to a greater consumption of this anticoagulant and anti-inflammatory protein $(19,20)$. However, when it was discovered that replacement therapy with recombinant human activated protein $\mathrm{C}$ had no significant effect on survival, neither from severe sepsis nor from ARDS, the interest in protein $C$ vanished $(27,28)$. Alterations in plasma coagulation and fibrinolysis have not yet been evaluated in view of the latest diagnostic criteria of ARDS that define "acute onset" as a condition developing within 1 week of a known insult, or a new or worsening respiratory failure (29).

We included patients during a 1-year period since some of the conditions with increased risk of ARDS, like influenza and pneumonia have their seasonal peaks. To detect a possible significance of plasma concentrations of TF, PAI-1, and t-PA, as diagnostic markers, we studied an ICU population, which had increased risk of ARDS and required mechanical ventilation for at least $24 \mathrm{~h}$. Several researchers have suggested a potential association between TF and the occurrence of ARDS. TF appears to play a pivotal role both in regulating endothelial permeability and in activating the external coagulation pathway in severe infections $(5,30,31)$. Moreover, investigators demonstrated higher plasma TF levels in parallel with lower PLTs and higher incidence of DIC in patients with ARDS, in whom the primary injury was trauma or sepsis (30). Recently, Xue and co-workers found that, patients with sepsis-induced ARDS had significantly higher levels of TF on the day of admission as compared with non-ARDS patients. The AUC for the diagnosis sepsis-induced ARDS was 0.749 . These investigators concluded that TF is an independent predictor of 30-day mortality in patients with severe sepsis (OR 1.41; 

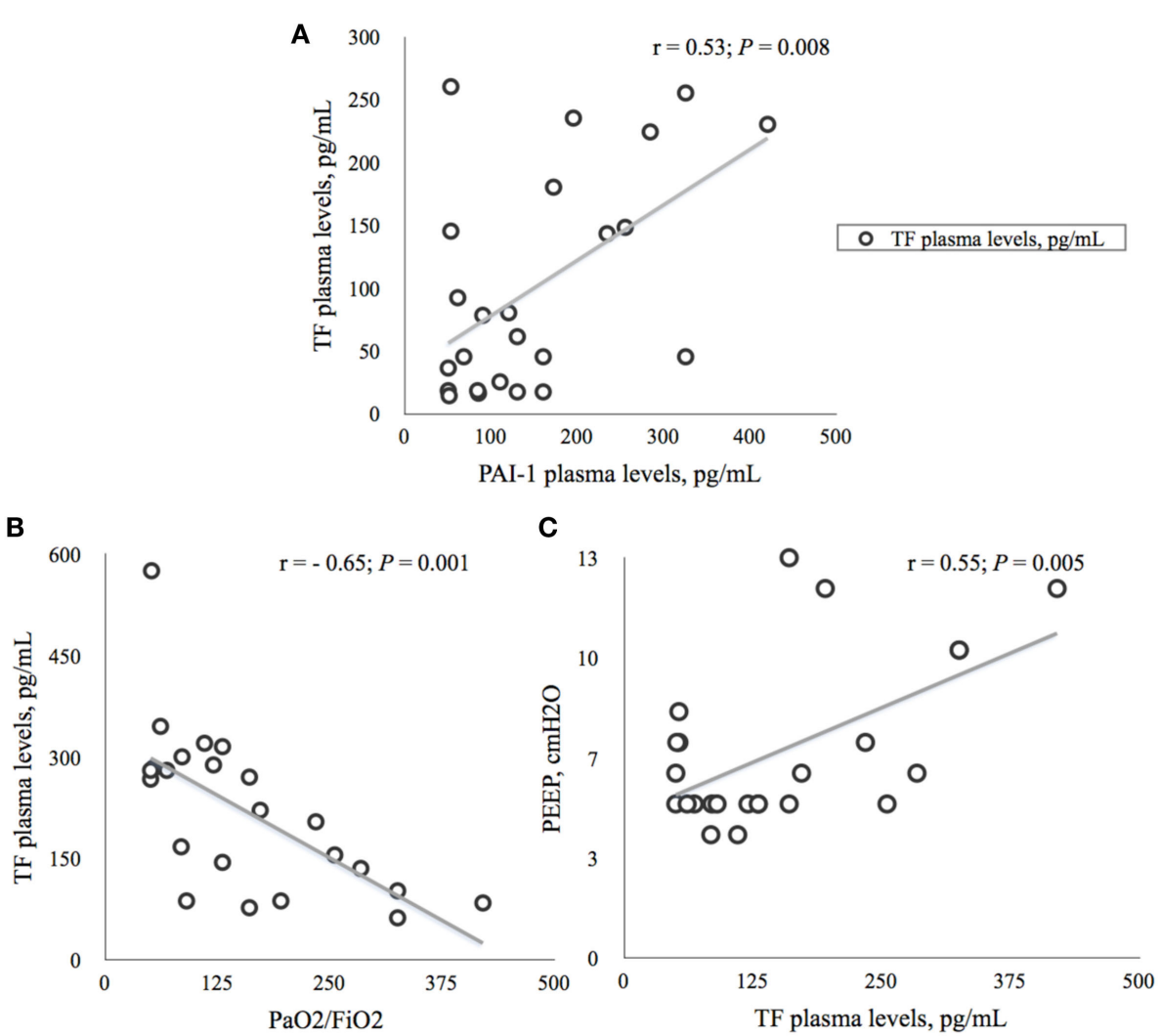

FIGURE 3 | Correlations between tissue factor (TF) and plasminogen activator inhibitor-1 (Al-1) and ventilation parameters on the seventh day ( $\left.T_{7}\right)$ of ICU stay. (A) Relationship between plasma concentrations of TF and PAl-1 in 24 ICU patients. Data presented as Spearman's correlation coefficient (r) with $p$-value. $\mathrm{TF}$, tissue factor; PAl-1, plasminogen activator inhibitor; pg/ml, picogram per milliliter. (B). Relationship between plasma concentrations of $\mathrm{TF}$ and $\mathrm{PaO}_{2} / \mathrm{FiO} \mathrm{O}_{2}$ in 24 ICU patients. Data presented as Spearman's correlation coefficient $(r)$ with $p$-value. $\mathrm{PaO}_{2}$, arterial oxygen partial pressure; $\mathrm{FiO}_{2}$, fraction of inspired oxygen; TF, tissue factor; pg/ml, picogram per milliliter. (C) Relationship between plasma concentrations of TF and level of PEEP in 24 ICU patients. Data presented as Spearman's correlation coefficient with $p$-value. PEEP, positive end-expiratory pressure; $\mathrm{cmH}_{2} \mathrm{O}$, centimeter of water; TF, tissue factor; pg/ml, picogram per milliliter.

95\% CI 1.24-1.69) with AUC 0.718 (5). Consistent with the latter workers, we showed that TF increased significantly the seventh day after inclusion in the ARDS group as compared with nonARDS patients (Figure 2A). A ROC curve analysis displayed a sensitivity of $71 \%$ with AUC of $0.78, p=0.02$, which indicates a fair test value in favor of diagnosing ARDS, according to the published criteria $(2,5)$. However, we did not observe any correlation between TF and DIC, since none of our patients was diagnosed with that condition. In contrast, TF plasma levels correlated moderately with ventilation parameters (Figures 3B,C), most likely, because of a high proportion of patients with pneumonia (42\%) in the ARDS group. Therefore, we consider TF to be a tool for supporting ARDS diagnosis, but without any predictive value since the increase in plasma concentration occurred after 1 week of ICU stay.

In the present study, PAI-1 plasma concentration also increased significantly in ARDS patients on the seventh day of stay in ICU (Figure 2B). The rise in PAI-1 correlated slightly with a parallel increase in TF (Figure 3A) and ROC curve analysis (Figure 4) revealed a sensitivity of $64 \%$ with AUC of $0.775 ; p=0.024$, which indicates a somewhat poorer test in favor of diagnosing ARDS, as compared with the corresponding test of TF. Previous investigators have noticed that ARDS patients exhibit early elevation of PAI-1 activity in both plasma and air spaces in concert with a decrease in alveolar fibrinolysis (32, 33). Thus, El Solh and co-workers showed that tracheal aspirate PAI-1 antigen levels, sampled $8 \mathrm{~h}$ after witnessed aspiration, increased by a fivefold in those who progressed to ARDS, as compared with those with uncomplicated aspiration pneumonitis (34). The reason for the discrepancy between our results and those of the latter researchers, aside from different analytic techniques, could be that changes in tracheal aspirate PAI-1 antigen concentration occurs earlier as compared to the changes in plasma concentration (32).

Lately, Sapru and co-workers demonstrated in pediatric patients, that increased plasma concentrations of PAI-1, as determined the day after diagnosing ARDS, were associated with increased mortality and fewer ventilator-free days until day 28 (24). Furthermore, investigators who showed that plasma concentrations of PAI-1 increased from days 0 to 3 after diagnosing ARDS, predicted death with an odds ratio of $1.66(p=0.006)(20)$. Moreover, a prospective study of 50 patients with ARDS at an early stage showed significantly higher PAI-1 levels in those who died as compared with those who survived (19). However, we 


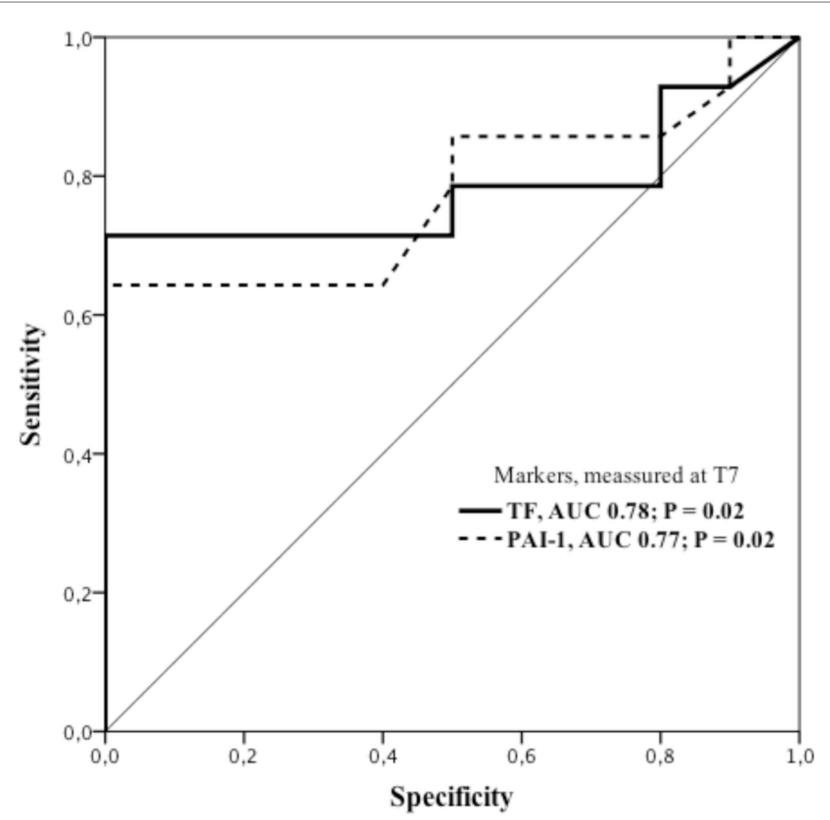

FIGURE 4 | ROC curves showing sensitivity and specificity of tissue factor and plasminogen activator inhibitor-1 for ARDS diagnoses on the seventh day $\left(\boldsymbol{T}_{\mathbf{7}}\right)$ of ICU stay. ARDS group displaying $71 \%$ sensitivity and $100 \%$ specificity for association with plasma concentration of TF. Cut off value: $145 \mathrm{pg} / \mathrm{ml}$; AUC 0.782; $p=0.021$; 95\%Cl: 0.586-0.979. Correspondingly, plasma concentration of PAl-1 showed sensitivity and specificity of 64 and $100 \%$, respectively, and a cut off value of $117.5 \mathrm{pg} / \mathrm{ml}$; AUC $0.775 ; p=0.024 ; 95 \% \mathrm{Cl}: 0.584-0.966$. AUC, area under the curve; 95\% Cl, 95\% confidence interval; PAl-1, plasminogen activator inhibitor-1; $\mathrm{ROC}$, receiver operating characteristics; TF, tissue factor.

confirmed none of the latter findings in this pilot study, which was underpowered for such investigations.

As an acute-phase protein, the serine protease inhibitor PAI-1 binds covalently to $\mathrm{t}-\mathrm{PA}$, reduces formation of plasmin, and impedes fibrinolytic bleeding. Moreover, recent experimental studies indicate that PAI-1 also plays a role in upregulating inflammation after exposure to lipopolysaccharides or avian flu virus in conditions like ARDS $(35,36)$. However, in the present study, when we compared the ARDS group with non-ARDS patients, we found no significant differences in t-PA levels during the first week after inclusion. Thus, whether t-PA is a predictive marker of ARDS is still not concluded, but this hypothesis was not supported in the present prospective pilot study.

We admit that our study has several limitations because of the small number of patients included. For this reason, we could not expect to find significant differences between groups neither in ICU stay and mechanical ventilation, nor in mortality rates. Despite the fact that we found a lower plasma concentration of t-PA at $T_{3}$ in ARDS survivors in comparison with non-survivors, the test had a power of only $60 \%$ due to low sample size. However, by keeping the ratio between the groups unchanged, an increase in sample size to 14 survivors and 6 non-survivors, would give a significant difference with a power of $80 \%$. Correspondingly, to reach a significant intergroup difference in t-PAI with the same power at $T_{7}$, would require a total sample size of 317 patients. Therefore, due to low sample sizes, we were not able to strengthen our findings by means of multivariate analysis. Since mean time to diagnosing ARDS was on the average 3 days, we also admit as a limitation of any predictive role, the fact that most of the patients already had ARDS at the time of plasma sampling at $T_{3}$, whereas significant changes in TF and PAI- 1 occurred first at $T_{7}$. Nevertheless, our observations indicate a role for TF and PAI-1 as tools for supporting ARDS diagnosis, although significant changes occurred relatively late in the evolution of disease.

We also denote as a weakness of this study that the ARDS patients incorporated many serious risk factors and comorbidities when admitted to the ICU. Therefore, those who were included had different lengths of hospital stays before admission to the ICU. However, the prior history was not an issue for evaluation in the present study.

According to ARDS definition (2), we should be able to identify patients with mild ARDS that potentially correlates with a better prognosis. However, besides a low number of patients included, we did not follow oxygenation variables often enough to be able to evaluate them properly against changes in ventilator settings, or other variables on an hourly basis. For the same reason, changes in concentrations of biomarkers that might have taken place, between the days of plasma sampling remain undiscovered. We also realize that sampling of bronchoalveolar lavage fluid, in parallel with the plasma samples, could have strengthened the findings, and maybe enabled the discovery of changes in biomarkers at an earlier time point.

A limitation is also the course of ARDS development that may influence the changes in biomarkers and outcome significantly. We included only patients with high risk of developing ARDS, but even among them, particularly patients with severe pneumonia have a poor prognosis in comparison with victims of multi-trauma and/or those undergoing massive blood transfusions. Finally, due to limited sample sizes, the findings of this pilot study need confirmation in a larger randomized controlled multicenter trial.

\section{CONCLUSION}

Fourteen of the 24 patients on mechanical ventilation whom we suspected to be at increased risk, developed ARDS on the average 3 days after admission to the ICU. Activation of the coagulation and the fibrinolytic systems in terms of increased plasma levels of TF and PAI-1 occurred on the seventh day of ICU stay in patients diagnosed with ARDS, thus indicating a supportive diagnostic role for these biomarkers. Our findings need confirmation in a larger randomized controlled multicenter trial.

\section{AUTHOR CONTRIBUTIONS}

All authors have contributed substantially to the study design, analysis and interpretation of data, and drafting or revising the article. AO, MS, and OS conceived the study. MS was responsible for data collection and informed the patients. IV, AS, IJ, and JS performed data analysis, $\mathrm{AO}$ and MS made interpretation of data. $\mathrm{AO}$ and LB drafted and revised the manuscript. All authors read and approved the final manuscript. 


\section{ACKNOWLEDGMENTS}

The authors thank Senior Engineer Timofey Kondratiev, $\mathrm{MD}, \mathrm{PhD}$, Anesthesia and Critical Care Research Group, The Department of Clinical Medicine, Faculty of Health Sciences, UiT, The Arctic University of Norway, 9037 Tromsø, Norway, for skilled technical assistance. The authors are also indebted to Dr. IJ, Head of Immunology Center, Pauls Stradins Clinical

\section{REFERENCES}

1. Donahoe M. Acute respiratory distress syndrome: a clinical review. Pulm Circ (2011) 1(2):192-211. doi:10.4103/2045-8932.83454

2. Ferguson ND, Fan E, Camporota L, Antonelli M, Anzueto A, Beale R, et al. The Berlin definition of ARDS: an expanded rationale, justification, and supplementary material. Intensive Care Med (2012) 38(10):1573-82. doi:10.1007/ s00134-012-2682-1

3. Terpstra ML, Aman J, van Nieuw Amerongen GP, Groeneveld AB. Plasma biomarkers for acute respiratory distress syndrome: a systematic review and meta-analysis*. Crit Care Med (2014) 42(3):691-700. doi:10.1097/01. ccm.0000435669.60811.24

4. Fanelli V, Vlachou A, Ghannadian S, Simonetti U, Slutsky AS, Zhang H. Acute respiratory distress syndrome: new definition, current and future therapeutic options. J Thorac Dis (2013) 5(3):326-34. doi:10.3978/j. issn.2072-1439.2013.04.05

5. Xue M, Sun Z, Shao M, Yin J, Deng Z, Zhang J, et al. Diagnostic and prognostic utility of tissue factor for severe sepsis and sepsis-induced acute lung injury. J Transl Med (2015) 13:172. doi:10.1186/s12967-015-0518-9

6. Ferguson ND, Frutos-Vivar F, Esteban A, Gordo F, Honrubia T, Penuelas $\mathrm{O}$, et al. Clinical risk conditions for acute lung injury in the intensive care unit and hospital ward: a prospective observational study. Crit Care (2007) 11(5):R96. doi:10.1186/cc6113

7. Iscimen R, Cartin-Ceba R, Yilmaz M, Khan H, Hubmayr RD, Afessa B, et al. Risk factors for the development of acute lung injury in patients with septic shock: an observational cohort study. Crit Care Med (2008) 36(5):1518-22. doi:10.1097/CCM.0b013e31816fc2c0

8. Kuzkov VV, Kirov MY, Sovershaev MA, Kuklin VN, Suborov EV, Waerhaug K, et al. Extravascular lung water determined with single transpulmonary thermodilution correlates with the severity of sepsis-induced acutelung injury. Crit Care Med (2006) 34(6):1647-53. doi:10.1097/01.CCM.0000218817.24208.2E

9. Rubenfeld GD, Caldwell E, Peabody E, Weaver J, Martin DP, Neff M, et al. Incidence and outcomes of acute lung injury. N Engl J Med (2005) 353(16):1685-93. doi:10.1056/NEJMoa050333

10. Linko R, Okkonen M, Pettila V, Perttila J, Parviainen I, Ruokonen E, et al. Acute respiratory failure in intensive care units. FINNALI: a prospective cohort study. Intensive Care Med (2009) 35(8):1352-61. doi:10.1007/ s00134-009-1519-z

11. Bellani G, Laffey JG, Pham T, Fan E, Brochard L, Esteban A, et al. Epidemiology, patterns of care, and mortality for patients with acute respiratory distress syndrome in intensive care units in 50 countries. JAMA (2016) 315(8):788-800. doi:10.1001/jama.2016.0291

12. Irish Critical Care Trials Group. Acute lung injury and the acute respiratory distress syndrome in Ireland: a prospective audit of epidemiology and management. Crit Care (2008) 12(1):R30. doi:10.1186/cc6808

13. Bernard GR, Artigas A, Brigham KL, Carlet J, Falke K, Hudson L, et al. The American-European Consensus Conference on ARDS. Definitions, mechanisms, relevant outcomes, and clinical trial coordination. Am J Respir Crit Care Med (1994) 149(3 Pt 1):818-24. doi:10.1164/ajrccm.149.3.7509706

14. Villar J, Blanco J, Kacmarek RM. Current incidence and outcome of the acute respiratory distress syndrome. Curr Opin Crit Care (2016) 22(1):1-6. doi:10.1097/MCC.0000000000000266

15. Gong MN, Thompson BT. Acute respiratory distress syndrome: shifting the emphasis from treatment to prevention. Curr Opin Crit Care (2016) 22(1):21-37. doi:10.1097/MCC.0000000000000275

16. Rodrigues CD, Oliveira RA, Soares SM, Figueiredo LC, Araujo S, Dragosavac D. Lung injury and mechanical ventilation in cardiac surgery:
University Hospital, and Professor Dr. Med. AS, Department of biochemistry, Rigas Stradins University for sharing their expertise.

\section{FUNDING}

The study was supported by grant ZP 10/2013 from Rigas Stradins University.

a review. Rev Bras Ter Intensiva (2010) 22(4):375-83. doi:10.1590/S0103507X2010000400011

17. Jozwiak M, Teboul JL, Monnet X. Extravascular lung water in critical care: recent advances and clinical applications. Ann Intensive Care (2015) 5(1):38. doi:10.1186/s13613-015-0081-9

18. Brower RG, Matthay MA, Morris A, Schoenfeld D, Thompson BT, Wheeler A. Ventilation with lower tidal volumes as compared with traditional tidal volumes for acute lung injury and the acute respiratory distress syndrome. The acute respiratory distress syndrome network. N Engl J Med (2000) 342(18):1301-8. doi:10.1056/NEJM200005043421801

19. McClintock D, Zhuo H, Wickersham N, Matthay MA, Ware LB. Biomarkers of inflammation, coagulation and fibrinolysis predict mortality in acute lung injury. Crit Care (2008) 12(2):R41. doi:10.1186/cc6846

20. Ware LB, Matthay MA, Parsons PE, Thompson BT, Januzzi JL, Eisner MD, et al. Blood Institute Acute Respiratory Distress Syndrome Clinical Trials N: pathogenetic and prognostic significance of altered coagulation and fibrinolysis in acute lung injury/acute respiratory distress syndrome. Crit Care Med (2007) 35(8):1821-8. doi:10.1097/01.CCM.0000221922.08878.49

21. Idell S, Koenig KB, Fair DS, Martin TR, McLarty J, Maunder RJ. Serial abnormalities of fibrin turnover in evolving adult respiratory distress syndrome. Am J Physiol (1991) 261(4 Pt 1):L240-8.

22. Prabhakaran P, Ware LB, White KE, Cross MT, Matthay MA, Olman MA Elevated levels of plasminogen activator inhibitor-1 in pulmonary edema fluid are associated with mortality in acute lung injury. Am J Physiol Lung Cell Mol Physiol (2003) 285(1):L20-8. doi:10.1152/ajplung.00312.2002

23. Brun-Buisson C, Minelli C, Bertolini G, Brazzi L, Pimentel J, Lewandowski $\mathrm{K}$, et al. Epidemiology and outcome of acute lung injury in European inten sive care units. Results from the ALIVE study. Intensive Care Med (2004) 30(1):51-61. doi:10.1007/s00134-003-2136-x

24. Sapru A, Curley MA, Brady S, Matthay MA, Flori H. Elevated PAI-1 is associated with poor clinical outcomes in pediatric patients with acute lung injury. Intensive Care Med (2010) 36(1):157-63. doi:10.1007/s00134-009-1690-2

25. Altman DG. Statistics in the medical literature: 3. Stat Med (1999) 18(4): 487-90. doi:10.1002/(SICI)1097-258(19990228)18:4<487::AID-SIM106>3.3. $\mathrm{CO} ; 2-\mathrm{A}$

26. Yan SB, Helterbrand JD, Hartman DL, Wright TJ, Bernard GR. Low levels of protein $\mathrm{C}$ are associated with poor outcome in severe sepsis. Chest (2001) 120(3):915-22. doi:10.1378/chest.120.3.915

27. Liu KD, Looney MR, Matthay MA. Inhaled activated protein C: a novel therapy for acute lung injury? Crit Care (2009) 13(3):150. doi:10.1186/cc7869

28. Angus DC. Drotrecogin alfa (activated)...a sad final fizzle to a roller-coaster party. Crit Care (2012) 16(1):107. doi:10.1186/cc11152

29. Force ADT, Ranieri VM, Rubenfeld GD, Thompson BT, Ferguson ND, Caldwell E, et al. Acute respiratory distress syndrome: the Berlin definition. JAMA (2012) 307(23):2526-33. doi:10.1001/jama.2012.5669

30. Gando S, Nanzaki S, Morimoto Y, Kobayashi S, Kemmotsu O. Systemic activation of tissue-factor dependent coagulation pathway in evolving acute respiratory distress syndrome in patients with trauma and sepsis. J Trauma (1999) 47(4):719-23. doi:10.1097/00005373-199910000-00017

31. van der Poll T. Tissue factor as an initiator of coagulation and inflammation in the lung. Crit Care (2008) 12(Suppl 6):S3. doi:10.1186/cc7026

32. Determann RM, Millo JL, Garrard CS, Schultz MJ. Bronchoalveolar levels of plasminogen activator inhibitor-1 and soluble tissue factor are sensitive and specific markers of pulmonary inflammation. Intensive Care Med (2006) 32(6):946-7. doi:10.1007/s00134-006-0167-9

33. Idell S, Peters J, James KK, Fair DS, Coalson JJ. Local abnormalities of coagulation and fibrinolytic pathways that promote alveolar fibrin deposition 
in the lungs of baboons with diffuse alveolar damage. J Clin Invest (1989) 84(1):181-93. doi:10.1172/JCI114217

34. El Solh AA, Bhora M, Pineda L, Aquilina A, Abbetessa L, Berbary E. Alveolar plasminogen activator inhibitor-1 predicts ARDS in aspiration pneumonitis. Intensive Care Med (2006) 32(1):110-5. doi:10.1007/s00134-005-2847-2

35. Wang ZH, Ren WY, Zhu L, Hu LJ. Plasminogen activator inhibitor-1 regulates LPS induced inflammation in rat macrophages through autophagy activation. ScientificWorldJournal (2014) 2014:189168. doi:10.1155/2014/189168

36. Ma J, Sun Q, Mi R, Zhang H. Avian influenza A virus H5N1 causes autophagy-mediated cell death through suppression of mTOR signaling. J Genet Genomics (2011) 38(11):533-7. doi:10.1016/j.jgg.2011.10.002
Conflict of Interest Statement: The authors declare that the research was conducted in the absence of any commercial or financial relationships that could be construed as a potential conflict of interest.

Copyright $\odot 2016$ Ozolina, Sarkele, Sabelnikovs, Skesters, Jaunalksne, Serova, Ievins, Bjertnaes and Vanags. This is an open-access article distributed under the terms of the Creative Commons Attribution License (CC BY). The use, distribution or reproduction in other forums is permitted, provided the original author(s) or licensor are credited and that the original publication in this journal is cited, in accordance with accepted academic practice. No use, distribution or reproduction is permitted which does not comply with these terms. 